\title{
Molecular Epidemiologic Typing Systems of Bacterial Pathogens: Current Issues and Perpectives
}

\begin{abstract}
Marc J Struelens
Service de Microbiologie, Hôpital Erasme and Unité d'Epidémiologie des Maladies Infectieuses, Ecole de Santé Publique, Université Libre de Bruxelles 808, Route de Lennik 1070 Bruxelles, Belgium

The epidemiologic typing of bacterial pathogens can be applied to answer a number of different questions: in case of outbreak, what is the extent and mode of transmission of epidemic clone $(s)$ ? In case of long-term surveillance, what is the prevalence over time and the geographic spread of epidemic and endemic clones in the population? A number of molecular typing methods can be used to classify bacteria based on genomic diversity into groups of closely-related isolates (presumed to arise from a common ancestor in the same chain of transmission) and divergent, epidemiologically-unrelated isolates (arising from independent sources of infection). Ribotyping, IS-RFLP fingerprinting, macrorestriction analysis of chromosomal DNA and PCR-fingerprinting using arbitrary sequence or repeat element primers are useful methods for outbreak investigations and regional surveillance. Library typing systems based on multilocus sequence-based analysis and strain-specific probe hybridization schemes are in development for the international surveillance of major pathogens like Mycobacterium tuberculosis. Accurate epidemiological interpretation of data obtained with molecular typing systems still requires additional research on the evolution rate of polymorphic loci in bacterial pathogens.
\end{abstract}

Key words: bacterial typing - DNA polymorphism - pulsed-field gel electrophoresis - ribotyping - RFLP typing PCR fingerprinting - randomly amplified polymorphic DNA (RAPD) - cross-infection - epidemiology surveillance

\section{WHY DO WE NEED EPIDEMIOLOGIC TYPING?}

Epidemiologic typing systems can be used for outbreak investigations, to confirm and delineate the patterns of transmission of one or more epidemic clone(s), to test hypotheses about the sources and vehicles of transmission of these clones and to monitor the reservoirs of epidemic organisms. Typing also contributes to epidemiologic surveillance and evaluation of control measures, by documenting the prevalence over time and circulation of epidemic clones in infected populations. Clearly, different requirements will be needed for these distinct applications (Maslow \& Mulligan 1996, Struelens et al. 1996).

The basic premise of epidemiologic typing is that isolates of an infectious agent that are part of the same chain of transmission are clonally related, that is the progeny of the same ancestor cell. Extensive genomic and phenotype diversity exists within populations of microbial pathogens of the same species. This diversity reflects the evolutionary divergence arising from mutations and gene

Fax: +32.555.64.59. E-mail: marc.struelens@ulb.ac.be Received 15 June 1998 Accepted 30 July 1998 flux. Clonally related isolates exhibit significantly more similar characters than unrelated isolates. These distinctive characters, called epidemiological markers, are scored by typing systems which are designed to optimize discrimination between epidemiologically related and unrelated isolates of the pathogen of interest (Maslow \& Mulligan 1996, Struelens et al. 1996). The threshold of marker similarity used for definition of a clone need to be adjusted to the species studied, the typing system used, the environmental selective pressure and the time and space scale of the study (Tibayrenc 1995, Struelens et al. 1996). Mutation rate and gene flux vary between species, pathovars and environments. In vivo micro-evolution of most pathogens remains poorly understood. Subclonal evolution and emergence of variants that occur in individual hosts or during prolonged transmission can be recognized by several high resolution molecular typing systems, like, for instance, macrorestriction analysis by pulsed-field gel electrophoresis (Struelens et al. 1993, 1996).

\section{CURRENT TECHNOLOGIES: HOW WELL DO THEY} FULFILL OUR NEEDS?

In recent years, the development and extensive use of high resolution molecular typing systems based on direct analysis of genomic polymorphism have greatly improved the understanding of the 
epidemiology of infectious diseases (Maslow \& Mulligan 1996, Struelens et al. 1996). However, the rapid diversification and incomplete comparative evaluation of these methods leave the microbiologist and the epidemiologist faced with a number of questions dealing with selection of the appropriate typing system(s) for solving a particular problem, as well as a lack of consensus about interpretation and communication of results.

Several criteria are proposed for evaluating the performance of typing systems (Maslow \& Mulligan 1996, Struelens et al. 1996). These criteria include: typeability, reproducibility, stability, discriminatory power, and epidemiologic concordance. Typeability refers to the proportion of isolates that can be scored in the typing system and assigned a type, ideally all isolates. Reproducibility refers to the ability of the typing system to assign the same type on repeat testing of the same strain. Stability is the biological feature of clonally derived isolates to express constant markers over time and generations. The stability of markers may be acceptable even in the presence of variation, provided that the typing system enables recognition of clonal relatedness and does not lead to misclassification of subclonal variants as epidemiologically unrelated. Discriminatory power is a key characteristic of typing systems, because it conditions the probability that isolates sharing identical or closely-related types are truly clonal and part of the same chain of transmission. Discriminating power can be calculated based on Simpson's index of diversity. Ideally, the index, based on testing a large number of epidemiologically unrelated isolates, should equal 1 . In other words, each independent isolate should be sufficiently different to be assigned to a distinct clone. In practice, a typing system, or combination of systems, displaying a discrimination index greater than 0.95 is acceptable. This level of discrimination corresponds to a $5 \%$ probability of erroneously assigning independent isolates to the same clone. Epidemiologic concordance is the capacity of a typing system to correctly classify into the same clone all epidemiologically related isolates from a well-described outbreak. Additional comparative studies are needed to establish the relative value of systems currently used for typing microbial pathogens. Moreover, there are important variations in the performance of a given method depending on the species and on modifications of the procedure as applied by different investigators.

In addition to its intrinsic performance when applied to a particular microbial pathogen, a typing system should have practical advantages. Versatility, or the ability to type any pathogen, given minor modifications of the method, is an impor- tant advantage for the study of nosocomial infections. Other practical aspects of typing systems include ease of performance and ease of result interpretation, as well as cost and availability of reagents and equipment. Moreover, results should be obtained rapidly enough to be useful in making decisions about management of an outbreak. Infection control problems which require rapid typing data include confirmation that an outbreak is occurring and identification of carriers of the epidemic clone to implement isolation precautions or decolonization therapy. Because there is no optimal typing system that meets all the above requirements, it is as a rule necessary to use a combination of systems. Rapid screening systems can be used initially for preliminary assessment of clonality. Confirmation can be obtained subsequently, if required, by using more reliable but less efficient typing systems. Recent reviews have proposed "optimal" first pass and alternate methods as well guidelines for interpreting differences for a typing number of bacteria when faced with the need to investigate outbreaks (Maslow \& Mulligan 1996, Struelens et al. 1996, Tenover et al. 1997).

Methods that index chromosomal DNA polymorphism are the best options for comparative typing of most bacteria, especially nosocomial pathogens (Tenover et al. 1997). Good resolution of genomic restriction fragment length polymorphisms (RFLP) analysis is obtained by: (i) transfer of restriction fragments onto membranes, followed by Southern-blot hybridization with DNA probes, and/ or (ii) use of endonucleases that have infrequent (<30) recognition sites in the chromosome, followed by separation of these macrorestriction fragments by pulsed-field gel electrophoresis (PFGE). Different types of nucleic acid probes are used for typing: (i) genes encoding metabolic, virulence or resistance functions; (ii) multicopy elements, including insertion sequences and transposons, and (iii) rRNA or rDNA sequences (ribotyping). Southern blot analysis of gene polymorphism was found moderately discriminating but highly reproducible and stable. Examples include the mec determinant for discriminating strains of methicillin-resistant Staphylococcus aureus and the exotoxin A probe for typing Pseudomonas aeruginosa strains from cystic fibrosis patients. IS-fingerprinting, or Southern blot analyses by using insertion sequences as probes, provides a very reproducible and highly discriminating typing tool. Discrimination is related to the presences of multiple copies of these elements at diverse locations in the chromosome. Careful selection and optimization of probe sequence, restriction endonucleases, electrophoresis and hybridization conditions need to be developed for each species or pathovar to be typed. These 
techniques are not rapid and required specialized reagents and expertise. International standardization of technique, reagents, type strains and nomenclature was established by public health reference laboratories for IS6110 RFLP-fingerprinting of Mycobacterium tuberculosis, which integrates standard computer analysis of patterns and a common database, and is now widely applied for large scale surveillance of tuberculosis (Bauer et al. 1998).

Ribotyping is the most versatile and the most widely used strategy of Southern blot analysis of bacterial genome polymorphism. The evolutionary conservation of ribosomal RNA makes it applicable as a universal bacterial probe. Many important pathogens, including Enterobacteriaceae, Listeria, Pseudomonas sp. and staphylococci have more than five ribosomal operons and thus produce ribotype patterns of 5 to 15 bands. Ribotyping is a robust method that exhibits excellent reproducibility and stability, both in vitro and in vivo during the course of outbreaks. It is commercially available in a fully automated and well-standardized format. However, its discriminatory power is only moderate, at a level equal or inferior to that of multilocus enzyme electrophoresis. This is related to the fact that ribosomal operons cover less than $0.1 \%$ of chromosomal length and tend to cluster in one particular region of the genome. Discrimination of ribotyping depends on species and on choice and number of restriction endonucleases used. No consensus has been achieved on optimal procedure and no general rules are available for interpretation of technically problematic results, like weakly hybridizing fragments.

Macrorestriction analysis resolved by pulsedfield gel electrophoresis has recently emerged as a gold standard for genome fingerprinting of microbial pathogens (Maslow \& Muligan 1996, Tenover et al. 1997). Careful selection of low-frequency cleaving enzymes enables cutting the whole bacterial chromosome of any species into less than 30 fragments, typically 10 to $700 \mathrm{~kb}$ in size. Periodic change in the orientation of electric field during agarose electrophoresis, or pulsed field gel electrophoresis, allows separation and size determination of these macrorestriction fragments. With minor modifications in the selection of enzymes and "pulsing protocols", PFGE can be applied to any bacterium or yeast. Although direct probing of recognition sequences by rare cutters detects variation in less than $0.01 \%$ of the chromosome, large size rearrangements, like sequence duplication, deletion, or insertion, will be readily detected as a shift in fragment size and/or number. In comparison with other typing methods, PFGE has shown equal or greater discriminatory power
(Maslow \& Muligan 1996). PFGE requires two to four days before results are available and specialized equipment that is more expensive than those required for PCR or Southern hybridization. Nevertheless, because of its superior versatility, reproducibility and resolution, genome macrorestriction analysis is currently a method of choice for typing a majority of nosocomial pathogens and some community-acquired pathogens (Tenover et al. 1997). The sensitivity of PFGE to detect genomic rearrangements makes appropriate interpretation of minor pattern differences a key to its correct application to outbreak investigations and surveillance studies. Interlaboratory standardization has not yet reached a sufficient level to allow the use of common type nomenclature or direct DNA pattern exchange.

In recent years, a number of PCR-based strategies have been developed for strain discrimination of microbial pathogens. In PCR-gene RFLP typing, a target sequence, 1 to $2 \mathrm{~kb}$ long and known to show polymorphism among strains of the species of interest, is amplified at high stringency. The amplified product is cut with restriction endonucleases and isolates are compared by RFLP pattern. The PCR-serotyping method takes advantages of the conserved sequences at each end of protein antigens genes, like flagellin and outer membrane proteins of Gram-negative pathogens, for amplification of allelic variant sequences encoding the central, antigenically variable portion of these proteins. The polymorphic alleles can be determined by amplicon characterization with suitable restriction endonucleases (PCR-RFLP serotyping (Harrington et al. 1997) or conformational analysis (e.g., single strand conformation analysis, or PCR-SSCP serotyping). The advantages of these methods over conventional serotyping include the unlimited availability of specific reagents, use of universal techniques and typeability of variant strains with cryptic antigens.

Although it is a rapid, simple and reproducible technique, PCR-RFLP typing has shown so far only moderate discrimination. Moreover, it can be biased either by mosaicism due to horizontal transfer, e.g., flagellin gene in Campylobacter jejuni (Harrington et al. 1997) or confounded by hypermutation rate at so-called contingency loci that undergo rapid rearrangements in response to environmental changes (e.g., protein A gene polymorphism in S. aureus (van Belkum et al. 1996).

As the logical next step, nucleotide sequencing of PCR-amplified genes is the most sensitive and accurate means of indexing localized DNA polymorphism for strain typing. However, the time required and cost of the procedure are currently limiting the use of this method which has been 
applied to type viruses such as hepatitis viruses and HIV, but also bacteria such as Streptococcus pyogenes (Perea Mejia et al. 1997). With the rapid progress of automated, high troughput methods like DNA chip technology (Chee et al. 1996), it is likely that PCR resequencing will be increasingly used for epidemiologic typing of viruses, bacteria and other pathogens in the years to come.

Arbitrarily-primed PCR (AP-PCR) typing, and similar methods like RAPD (random amplified polymorphic DNA) and DAF (DNA amplification fingerprinting), are based on low-stringency PCR amplification by using a single, 10 to 20 -mer primer of arbitrary sequence. In the early cycles of the PCR reaction, the primer anneals to multiple sequences with partial homology, and fragments of DNA lying within less than a few kb between annealing sites on opposite DNA strands are amplified. After additional cycles, a strain-specific array of amplified DNA segments of various sizes is obtained. This simple and rapid technique has been successfully applied to genotypic strain delineation and genetic population analysis of a broad range of microbial pathogens, including bacteria, fungi and protozoans. All isolates are typeable and no prior knowledge of target genome sequences is necessary. Discrimination is good and correlates well with other genotyping techniques. The discriminatory power is variable according to number and sequence of arbitrary primers and amplification conditions. In spite of its attractive efficiency, AP-PCR typing suffers from problems in reproducibility and from the lack of consensus rules for interpretation of pattern differences (Maslow \& Mulligan 1996, Struelens et al. 1996). A number of technical factors need to be strictly standardized for optimal reproducibility (Grundmann et al. 1997). Progress toward enhanced resolution and reproducibility of analysis of PCR products is achieved by incorporating fluorescent primers in the reaction and performing computer-analysis of amplimer patterns by an automated laser fluorescence detection system. In general, differences in protocols, equipment, or even the batch of reagent used result in different AP-PCR patterns, but the overall clustering and grouping of isolates into identical, similar, or divergent patterns is reproducible. This makes the method adequate for rapid comparative typing but less suitable for library typing in surveillance programs.

Repetitive element PCR (rep-PCR) typing consists of PCR amplification of spacer fragments lying between repeat motifs of the genome by use of two outwardly-directed primers at high stringency. Short, repetitive elements which have been successfully used as targets for rep-PCR typing include the repetitive extragenic palindromes (REPs), the enterobacterial repetitive intergenic consensus (ERIC) sequences, insertion sequences and other species-specific repeat elements (Maslow \& Mulligan 1996, Deplano et al. 1997). These repPCR strategies produce fewer amplified DNA fragments than AP-PCR, but can nevertheless provide good discriminatory power. Their major advantage is a better reproducibility as compared with APPCR analysis, which may enable their standardization for use as library typing systems.

Another set of innovative PCR-based strategy, which also appears to offer high resolution and good reproducibility, are the amplified fragment length polymorphism (AFLP) method (Vos et al. $1995)$ and infrequent restriction site amplification (IRS-PCR) (Mazurek et al. 1996). In these methods, a restriction-ligation step produces restricted genomic DNA fragments tagged with specially designed adapters. A set of different primers complementary to these adapters and adjacent nucleotides are then used to PCR amplify various parts of the tagged restriction fragments, thereby selectively highlighting a subset of restriction fragments. More studies are needed to determine the stability of these markers over time, establish criteria for interpretation of pattern differences and evaluate inter-laboratory reproducibility.

Finally, specialized genotyping schemes use reverse dot blot or line blot binary hybridization patterns of crude genomic DNA or amplified regions thereof with immobilized, clone-specific DNA probes. This method has been developed for typing of S. aureus (van Leeuwen et al. 1996) and M. tuberculosis (Kamerbeek et al. 1997). These library probe genotyping systems provide unambiguous, numeric clonal signatures that should be reproducible between laboratories. Inclusion of additional polymorphic sequences should increase the discrimination to the level needed for surveillance of major pathogens. The power of these genotypic hybridization schemes could be much enhanced by the use of high density DNA probe assays, as this technology currently allows parallel analysis of $10^{4}$ target sequences within a few hours.

\section{HOW TO INTERPRETE DIFFERENCES OBSERVED BETWEEN GENOTYPES ?}

We use molecular typing systems in epidemiologic studies to determine if isolates are clonally related and thus belong to the same chain of transmission. When a set of isolates show identical DNA banding patterns, this clue to clonality is proportional to the number of typing systems used and their discriminatory power. A problem arises when patterns are similar but not identical. What level of pattern similarity can be used to define clonally or epidemiologically related organisms? This level 
needs to be adjusted to the resolving power of the system used, the genomic plasticity of the organism under study and the time scale of the investigation. Suggested rules for interpretation of differences in PFGE patterns, as applied to outbreak investigations (Struelens et al. 1996, Tenover et al. 1997), relate the gradual increase in the number of restriction fragment mismatches with increasing number of genetic differences and with decreasing probability of epidemiologic relatedness. Calculation of restriction/hybridization pattern similarity coefficients and graphical display of pattern relatedness as dendrograms is also useful for interpretation, particularly for large scale studies (Struelens et al. 1996). Altough this quantitative analysis has been criticized as invalid for phylogenetic inferences, because DNA restriction fragment pattern variation is not due to independent events, it is supported by population analysis, e.g. of P. aeruginosa. Additional population genetic and micro-evolution studies are needed to provide a better understanding of the nature and frequency of molecular events giving rise to genomic polymorphisms exploited empirically by epidemiologists for strain typing (Tibayrenc 1995, Struelens et al. 1996)

Molecular typing systems are undergoing rapid technical improvements. Advances in the understanding of biological basis of microbial biodiversity at subspecies levels will improve the conceptual framework required for proper epidemiologic interpretation of typing results. Wider application of these systems should shed light to the epidemiology of hospital and community-acquired infections and, therefore, allow for more effective control and prevention strategies.

\section{REFERENCES}

Bauer J, Yang Z, Poulsen S, Andersen AB 1998. Results from 5 years of nationwide DNA fingerprinting of Mycobacterium tuberculosis complex isolates in a country with a low incidence of $M$. tuberculosis infection. J Clin Microbiol 36: 305-308.

Chee M, Yang R, Hubbell E, et al. 1996. Accessing genetic information with high-density DNA arrays. Science 274: 610-614.

Deplano A, Vaneechoutte M, Verschraegen G, Struelens MJ 1997. Typing of Staphylococcus aureus and Staphylococcus epidermidis strains by PCR analysis of inter-IS256 spacer length polymorphisms. J Clin Microbiol 35: 2580-2587.

Grundmann HJ, Towner KJ, Dijkshoorn L, et al. 1997. Multicenter study using standardized protocols and reagents for evaluation of reproductibility of PCRbased fingerprinting of Acinetobacter spp. J Clin Microbiol 35: 3071-3077.

Harrington CS, Thomson-Carter FM, Carter PE 1997. Evidence for recombination in the flagellin locus of
Campylobacter jejuni : implications for the flagellin gene typing scheme. J Clin Microbiol 35: 23862392.

Kamerbeek J, Schould L, Kolk A, et al. 1997. Simultaneous detection and strain differentiation of $\mathrm{Myco}$ bacterium tuberculosis for diagnosis and epidemiology. J Clin Microbiol 35: 907-914.

Maslow J, Mulligan ME 1996. Epidemiologic typing systems. Infect Control Hosp Epidemiol 17: 595604.

Mazurek GH, Reddy V, Marston BJ, Haas WH, Crawford JT 1996. DNA fingerprinting by infrequent-restriction-site amplification. J Clin Microbiol 34: 23862390.

Perea Mejia LM, Stockbauer KE, Pan X, Cravioto A, Musser JM 1997. Characterization of group A Streptococcus strains recovered from Mexican children with pharyngitis by automated DNA sequencing of virulence-related genes : unexpectedly large variation in the gene (sic) encoding a complement-inhibiting protein. J Clin Microbiol 35: 3220-3024.

Struelens MJ, and the Members of the European Study Group on Epidemiological Markers (ESGEM), of the European Society for Clinical Microbiology and Infectious Diseases (ESCMID) 1996. Consensus guidelines for appropriate use and evaluation of microbial epidemiologic typing systems. Clin Microbiol Infect 2: 2-11.

Struelens MJ, Schwam V, Deplano A, Baran D 1993. Genome macrorestriction analysis of diversity and variability of Pseudomonas aeruginosa strains infecting cystic fibrosis patients. J Clin Microbiol 31: 2320-2326.

Tenover FC, Arbeit RD, Goering RV, the Molecular Typing Working Group of the Society for Healthcare Epidemiology of America 1997. How to select and interpret molecular typing methods for epidemiological studies of bacterial infections: a review for healthcare epidemiologists. Infect Control Hosp Epidemiol 18: 426-439.

Tibayrenc M 1995. Population genetics of parasitic protozoa and other microorganisms. Advances Parasitol 136: 47-115.

van Belkum A, van Leeuwen W, Kaufmann ME, et al. 1998. Assessment of resolution and intercenter reproducibility of results of genotyping methicillinresistant Staphylococcus aureus by pulsed-field gel electrophoresis of $\mathrm{SmaI}$ macrorestriction fragments: a multicenter study. J Clin Microbiol 36: 1653-1659.

van Belkum A, Riewerts Eriksen N, et al. 1996. Are variable repeats in the spa gene suitable targets for epidemiological studies of methicillin-resistant Staphylococcus aureus strains? Eur J Clin Microbiol Infect Dis 15: 768-769.

van Leeuwen W, Sijmons M, Sluijs J, Verbrugh H, van Belkum A 1996. On the nature and use of randomly amplified DNA from Staphylococcus aureus. J Clin Microbiol 34: 2770-2777.

Vos P, Hogers R, Bleeker M, et al. 1995. AFLP: a new technique for DNA fingerprinting. Nucleic Acids Res 23: 4407-4014. 
